\title{
Estandarización de una técnica en el tiempo y su impacto en los resultados para hiperhidrosis palmar primaria
}

\author{
José Manuel Hernández G. ${ }^{1}$, Miguel Ángel Martínez A. , \\ Gustavo Salinas S. ${ }^{2}$ y Josefina Nodal O. ${ }^{1}$
}

\section{Our technique standardization on time and how impact over outcomes for primary palmar hiperhidrosis}

Introduction: Primary palmar hyperhidrosis is a frequent medical problem. Minimizing invasion, simplifying and standardizing technique means better results. Aim: To evaluate efficacy and safety with the application of our simplified and standardized technique, through its impact on trans and post-operative outcomes for primary palmar hyperhidrosis (PPH). Minimizing approach, simplifying and standardizing the technique should lead to better outcomes. Materials and Method: An observational study is carried out to evaluate the outcomes obtained with the application of our standardized technique for the treatment of PPH. The universe constituted by 359 patients treated between 2007 and 2011. Control up to 5 years was possible in 298, which constituted the definitive sample. Results: 298 patients (596 procedures) were analyzed. In the transoperative only 3 patients $(1 \%)$ report minor complication. Thoracic pain management was the greatest difficulty immediate to surgery, with $61.7 \%$ moderate pain and $15.2 \%$ severe. Satisfaction indexes in excellent range according to validated surveys. Outpatient management in $99 \%$. Discussion: Simple intubation avoids respiratory complications, suspensions, use of expensive resources or highly qualified personnel. Early pain relief, non-uses of pleural tubes or antibiotics allows rapid recovery and early discharge due to simplification and standardization of the technique. Conclusion: The use of simple endotraqueal tube and oxygenation in apnea, single port, without pleural drainage or antibiotics and ambulatory is a safe practice for endoscopic thoracic sympathectomy, once achieved in necessary training. Key words: thoracic sympathectomy; simplified surgical technique; standardization; ambulatory.

\section{Resumen}

Introducción: La hiperhidrosis palmar primaria es un problema médico frecuente. Minimizar la invasión, simplificar y estandarizar la técnica supone mejores resultados. Objetivo: Evaluar eficacia y seguridad con la aplicación de nuestra técnica simplificada y estandarizada, a través de su impacto en los resultados trans y postoperatorio para la hiperhidrosis palmar primaria (HPP). Materiales y Método: Se realiza estudio observacional que evalúa los resultados obtenidos con la aplicación de nuestra técnica estandarizada para el tratamiento de HPP. El universo está constituido por 359 pacientes intervenidos entre 2007 y 2011. El control hasta los 5 años fue posible en 298, los que constituyeron la muestra definitiva. Resultados: Se analizaron 298 pacientes (596 procedimientos). En el transoperatorio solo 3 pacientes (1\%) presentaron alguna complicación. El manejo del dolor torácico fue la mayor dificultad inmediata a la cirugía, con $61,7 \%$ de dolor moderado y $15,2 \%$ severo. Último control a 5 años con curación en el 99,7\% y una recidiva. Los índices de satisfacción se muestran en rango excelente según encuestas validadas. Manejo ambulatorio en 99\%. Discusión: Aplicar intubación endotraqueal simple permite extrapolar ventajas ya conocidas de esta técnica y menos complicaciones respiratorias, suspensiones, uso de recursos costosos o personal muy calificado. La combinación de simplificar aspectos quirúrgicos y anestésicos permitieron estandarizar y simplificar nuestra técnica y con ello: no necesitar drenajes pleurales, menos dolor, recuperación casi inmediata de la fisiología respiratoria y alta precoz. Conclusión: Combinar el empleo de tubo endotraqueal simple y oxigenación apneíca con minimizar la invasión quirúrgica: puerto único, sin drenaje pleural y manejo ambulatorio constituyen una práctica segura para la simpatectomía torácica endoscópica, con mejores resultados postoperatorios, una vez logrado en entrenamiento necesario.

Palabras clave: simpatectomía torácica; técnica quirúrgica simplificada; estandarización; ambulatorio.
'Universidad de Ciencias Médicas de La Habana, Centro Nacional de Cirugía de Mínimo Acceso. La Habana, Cuba.

${ }^{2}$ Clínica Avendaño. Lima, Perú.

Recibido el 21 de septiembre de 2018 y aceptado para publicación el 13 de marzo de 2019.

Correspondencia a: Dr. José Manuel Hernández G. josemhdezdr@gmail.com 


\section{Introducción}

Existe un gran interés e innumerables investigaciones en las 2 últimas décadas en relación al tratamiento de la hiperhidrosis palmar primaria. Preocupación de sociedades científicas, congresos mundiales y grandes avances en general, pero algunos aspectos no cuentan con suficiente evidencia ${ }^{1,2}$. La cirugía se presenta como la opción curativa, más allá del sudor compensatorio y otros efectos secundarios potenciales, pero se reportan diferencias tanto anestésicas como quirúrgicas ${ }^{3-6}$. En relación a tales controversias se generan las siguientes interrogantes que constituyen nuestro problema a investigar:

- Qué tipo de anestesia: ¿anestesia general con tubo endotraqueal simple y oxigenación en apnea es efectiva y segura para este procedimiento?

- Cómo crear el campo operatorio: ¿la combinación de la posición y el propio neumotórax que crea el paso del trocar, permiten un campo operatorio adecuado?

- A qué nivel y como tratar el nervio: ¿ECR3M y ECR4M según ISSS es efectivo y seguro para la variante palmar?

- Cómo tratar el nervio y con qué instrumental: ¿el hook y corriente monopolar es costo efectivo y seguro para tratar el nervio?

- Uso o no de antibióticos ¿cómo cirugía limpia que es, se puede manejar sin antibióticos?

- Uso de drenaje pleural ¿se pueden manejar estos pacientes de manera segura y efectiva sin necesidad de drenaje pleural?

- Control postoperatorio: ¿se puede incluir de manera segura este procedimiento en los protocolos de manejo ambulatorio?

Las respuestas tenderán a estandarizar el tratamiento quirúrgico y constituyen el problema de esta investigación ${ }^{7,8}$.

Nuestro equipo comenzó a trabajar en el tema en 2003. Evolucionamos con la época pasando de la anestesia general con tubo de doble luz al tubo endotraqueal simple con insuflación de $\mathrm{CO}_{2}$, hasta llegar a la oxigenación apnéica como elemento técnico fundamental en nuestra técnica actual estandarizada. La posición del paciente fue simplificándose del decúbito prono, al lateral, hasta la posición semisentada actual, bastante universalmente aceptada. El tratamiento del nervio simpático fue dirigido en sus inicios a la sección de ramas preganglionares. Se buscaba desde entonces disminuir el sudor compensatorio, pero las recidivas eran muy altas. Posteriormente realizamos simpatectomía (resección). La RSR2A y RSR3B (nomenclatura internacional exi- gida por ISSS International Society of Simpathethic Surgery) $)^{9}$. Los resultados eran excelentes; pero con la simple sección del nervio (ECR3M Y ECR4M) se comenzaron a lograr los mismos resultados de manera más sencilla, más segura y más rápida, completando así la triada de modificaciones técnicas: anestesia, posición y tratamiento del nervio. Esta estandarización permitió reducir considerablemente los tiempos quirúrgicos, la generalización de la técnica y manejo ambulatorio y otras ventajas.

A finales de 2006 ya nuestros resultados eran estadísticamente uniformes. Investigaciones en nuestro centro sugerían cumplida la curva de aprendizaje. Quedó en esta fecha protocolizada la técnica quirúrgica para simpatectomía en nuestra institución con los detalles mencionados.

El presenta trabajo tiene por objetivo evaluar los resultados a largo plazo de la técnica ECR3M y ECR4M según ISSS, usando oxigenación en apnea, sin drenaje pleural, puerto único y manejo ambulatorio, como elementos protocolizados de estandarización y simplificación.

\section{Materiales y Método}

Se realiza estudio observacional de corte longitudinal y retrospectivo que evalúa eficacia y seguridad con la aplicación de nuestra técnica simplificada y estandarizada, en el tratamiento de la hiperhidrosis palmar primaria.

En un corte a finales de 2006 existían estadísticas en nuestra institución que demostraban buenos resultados por conceptos de tiempo quirúrgico, complicaciones, alta hospitalaria y otros, con nuestra variante técnica en el manejo quirúrgico de estos pacientes. Los 359 pacientes operados entre enero de 2007 y diciembre de 2011 constituyeron el universo de estudio, considerando cumplida la curva de aprendizaje. La inclusión de pacientes obedeció un orden consecutivo, excluyendo solamente aquellos que en diciembre de 2011 no cumplían con el criterio de 5 años de control. Para esta fecha de corte final quedó una muestra definitiva de 298 pacientes.

Se mesuraron variables transoperatorias como tiempo quirúrgico, complicaciones (sangrado, parada cardiorrespiratoria, lesiones intraoperatorias), variables postoperatorias inmediatas como dolor, curación, complicaciones (neumotórax, hemotórax, sepsis), y postoperatorias a largo plazo como complicaciones, efectos secundarios, recidivas y satisfacción, que permitirían demostrar que es una técnica segura, confiable y ventajosa respecto a otras variantes como diferentes niveles de sección 
ganglionar, alternativas para tratar el nervio como el clipping u otros métodos, uso de multitrocar, uso de $\mathrm{CO}_{2}$ para crear campo operatorio o tubo endotraqueal de doble lumen entre otras tantas variantes usadas.

El cálculo de media, porcentajes y mediana fueron los análisis más utilizados para estas variables.

La técnica quirúrgica empleada simplifica el procedimiento en diferentes aspectos y tiempos de la técnica como sigue.

Posición del paciente: Semisentado, con ambos brazos en abducción $90^{\circ}$ respecto al tronco.

Anestesia: General con tubo endotraqueal simple y uso de oxigenación en apnea durante el acceso como sigue.

Abordaje: Bilateral en el mismo tiempo anestésico, puerto único y coordinada con anestesia de forma que el cirujano realiza incisión de piel de 7 $\mathrm{mm}$ en fosa axilar, túnel de 3 a $4 \mathrm{~cm}$ bajo pectoral mayor en dirección a línea media clavicular y se posiciona sobre borde superior de $3^{\text {ra }}$ costilla línea clavicular media o cercano a ella y en este punto se comunica con anestesia para desacoplar al paciente de la ventilación mecánica (al tiempo que se administra oxigenación $100 \%$ ) y solo en este momento el cirujano entra en cavidad con trocar romo.

Campo operatorio: La suma de no ventilación y por tanto no presión positiva intrapulmonar, más posición semisentado del paciente y la presión positiva que se produce al pasar el trocar (sin válvula), aún sin administrar $\mathrm{CO}_{2}$, facilita un colapso pulmonar excelente para realizar el procedimiento.

Tratamiento quirúrgico del nervio: Sección ECR3M Y ECR4M sobre costillas 2 y 3 visible (según ISSS International Society of Sympathetic Surgery) que se extiende lateralmente sobre la misma costilla unos $4 \mathrm{~cm}$ en busca de cortar cualquier rama de Kuntz que pudiera aparecer ${ }^{9}$.

Cierre de puerto sin drenaje: Este paso es clave para evitar neumotórax residual una vez que no dejamos sonda pleural. Nuevamente la coordinación con anestesia se impone. Concluido el proceder y coordinado con anestesia, se comienza a ventilar nuevamente y se da seguimiento bajo visión directa a la reexpansión pulmonar. Una vez el pulmón llega arriba y contacta pleural parietal al vértice, comenzamos a retirar el trocar lentamente con la óptica dentro del mismo. Este proceso se continúa bajo ventilación continua hasta lograr la triple imagen pulmón-pleura-trocar, o imagen de "labios" que se muestra en Figura 1. Esta es la confirmación de reexpansión $100 \%$ y en este punto anestesia indica la retirada, la cual se realiza en un movimiento ágil, al tiempo de la ventilación mantenida.
Postoperatorio: Fuerte manejo de dolor y educación permite alta a partir de $4 \mathrm{~h}$ y manejo ambulatorio sin sondas o antibióticos.

Es conocido un período "seguro" de tiempo con oxigenación apnéica, sin que esto implique riesgo al paciente. La duración según la literatura es de 30 a 40 min y durante el mismo se produce únicamente una hipercapnia permisiva, que se borra rápidamente una vez reiniciada la ventilación y sin ningún otro cambio asociado ${ }^{10,11}$. Nuestro protocolo institucional pauta 20 min máximo por mayor seguridad. Si se necesita más tiempo para el proceder, se acopla el tubo endotraqueal y se ventila hasta optimizar antes de continuar con el proceder.

\section{Resultados}

La muestra en estudio tuvo un comportamiento demográfico con predominio de pacientes jóvenes, habiendo 85 de cada 100 entre segunda y tercera década de la vida. El sexo femenino y la raza blanca con predominio según muestra con mayor detalle en la Figura 2.

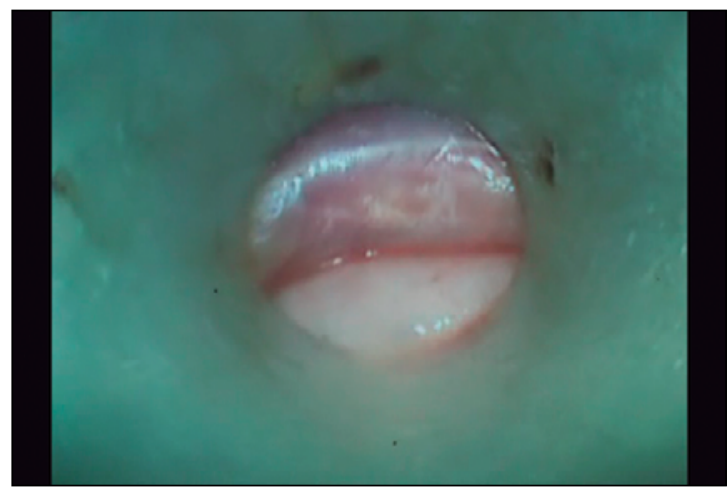

Figura 1. Triple imagen pulmón, pleura y trocar indica reexpansión pulmonar $100 \%$.

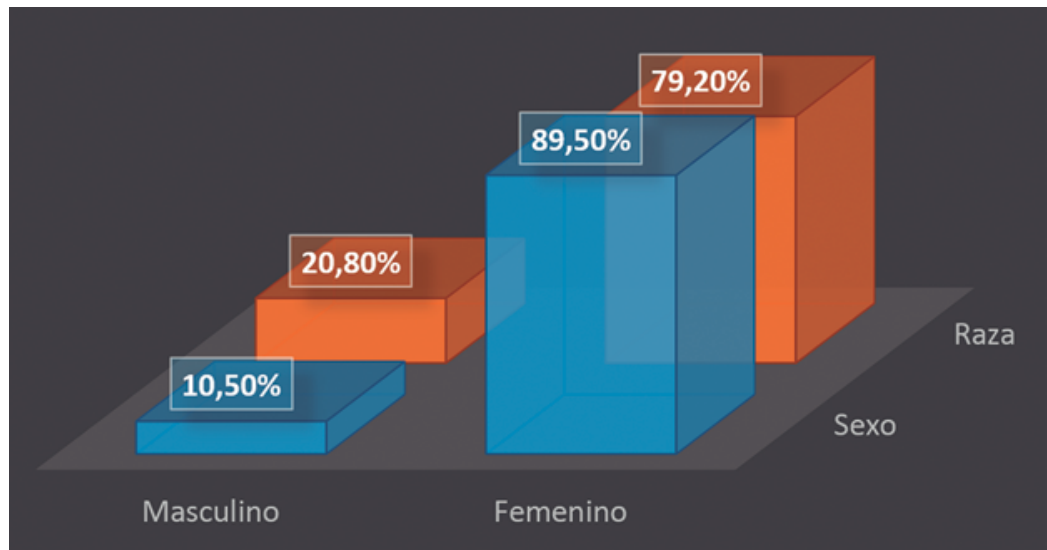

Figura 2. Distribución según sexo y raza. 
Durante el transoperatorio no se registró lesión intratorácica de ningún órgano. Tres pacientes (1,0\%) presentaron algún evento intraoperatorio que requirió alguna medida adicional. El primero, paciente masculino, obeso, por un sangrado moderado procedente de afluente venoso de la hemiácigos: un segundo paciente, masculino, 19 años, salud aparente, presentó parada cardiaca en asistolia que respondió rápidamente a reanimación y el tercero, femenino, 29 años, presentó adherencias intratorácicas gruesas y múltiples que impedían visualizar el nervio simpático.

La mediana del tiempo quirúrgico fue de $14 \mathrm{~min}$. Solo el $2 \%$ de los pacientes requirió 30 min o más de cirugía. Con un tiempo quirúrgico de 20 min se intervino al $31 \%$ de los operados y una mayoría del $67 \%$ consumió únicamente 10 min de cirugía.

El 99,7\% de la muestra se mantuvo estable durante todo el proceder, con la técnica anestésica de oxigenación apnéica. Saturación de oxígeno arterial periférica entre $98 \%$ y $100 \%$ y un valor medio del $\mathrm{CO}_{2}$ expirado de $60 \mathrm{mmHg}$ cuando más alto. Estabilidad hemodinámica y medio interno. Sin cambios significativos en monitoreo transoperatorio.

Tabla 1. Complicaciones que fueron rastreadas en el postoperatorio inmediato

\begin{tabular}{|lll|}
\hline Complicaciones inmediatas & & \\
Infección herida & $0 \%$ & $(0$ paciente $)$ \\
Dolor leve o moderado & $84,8 \%$ & $(253$ pacientes $)$ \\
Hemotórax & $0 \%$ & $(0$ paciente $)$ \\
Neumotórax laminar & $3,69 \%$ & $(11$ pacientes $) 100$ \\
Atelectasias segmentarias & $0 \%$ & $(0$ pacientes $)$ \\
\hline
\end{tabular}

Tabla 2. Efectos secundarios con mejoría estadísticamente significativa

\begin{tabular}{|llll|}
\hline Efectos secundarios & $\mathbf{1}$ año & $\mathbf{5}$ años & p valor \\
\hline Sudor compensatorio moderado* & $183(61,4 \%)$ & $118(39,5 \%)$ & \\
Sudor compensatorio severo* & $1(0,3 \%)$ & $1(0,3 \%)$ & \\
Sudoración gestatoria & $2(0,69 \%)$ & $2(0,69 \%)$ & \\
Mejoría plantar & $119(39,9 \%)$ & $52(17,4 \%)$ & \multirow{2}{*}{$\mathrm{p}=0,001$} \\
Resequedad de piel (manos) & $25(8,38 \%)$ & $5(1,6 \%)$ & \\
Claude Bernard Horner & $0(0 \%)$ & $0(0 \%)$ & \\
Desregulación de la temperatura & $0(0 \%)$ & $0(0 \%)$ & \\
Dolor torácico crónico & $0(0 \%)$ & $0(0 \%)$ & \\
\hline
\end{tabular}

En el postoperatorio inmediato se obtuvo $100 \%$ de curación al nivel palmar evaluado. El dolor torácico fue la variable de mayor positividad. El $100 \%$ de los operados presentaron algún grado de dolor. La tendencia fue al aumento de la intensidad en las primeras 48 a 72 h. Según la escala visual analógica EVA, la media de los operados presentó dolor moderado. En términos de porcentaje 23,1\% dolor leve (69 pacientes) y 61,7\% moderado (184 pacientes). El 15,2\% presentó dolor severo (45 pacientes). Otras complicaciones pesquizadas: neumotórax, hemotórax o sepsis se muestran en la Tabla 1.

A largo plazo, no se presentaron complicaciones. Para evaluar la eficacia y la seguridad de la simpatectomía se emplean universalmente las siguientes variables: 1) Recidivas, 2) Efectos Secundarios y 3) Nivel de Satisfacción. Recidivas o efectos secundarios no tienen ninguna relación con la técnica anestésica o la intubación. El nivel de satisfacción es un resultado mediado por la técnica en su conjunto.

En relación a la primera, solamente un paciente $(0,3 \%)$ de los 298 evaluados presentó recidiva de la sudación palmar a 5 años, resultando una eficacia de $99,7 \%$. Si bien con menor intensidad, el paciente optó por reoperarse.

El sudor compensatorio, el sudor gustatorio, y otros como síndrome de Claude Bernard Horner se comportaron según muestra la Tabla 2. Los resultados son comparables con la mayor evidencia disponible.

Series internacionales muestran resultados estadísticamente similares a nuestra investigación. Algunos ejemplos de estos resultados en la Tabla 3.

El nivel de satisfacción se evaluó con la encuesta sobre calidad de vida consensuada por un grupo de expertos internacionales en Congreso Latinoamericano de Cirugía de Tórax de México 2013: obtuvimos $92 \%$ de excelente y $5 \%$, con un $97 \%$ de pacientes con niveles de satisfacción postoperatorio entre bueno y excelente. Un $3 \%$ regular y un $1 \%$ con mal según la encuesta antes referida.

Se aplicaron paralelamente los test de IDARE para ansiedad y la prueba de Zung y Conie para depresión, al inicio y al control de los 5 años, ambos validados para nuestra población. Se pudo observar en ambos casos un mejoramiento significativo en la evaluación final. El comparativo se desarrolla en la Tabla 4.

El manejo postoperatorio de estos pacientes se realizó de forma ambulatoria con 244 egresos a las $4 \mathrm{~h}$ de operados para el $81,8 \%$, otros 51 operados a las $8 \mathrm{~h}$ para el $7,2 \%$ y solo 3 pasadas las $24 \mathrm{~h}$ lo que representa un $1 \%$. 
Tabla 3. Modificaciones introducidas y su impacto

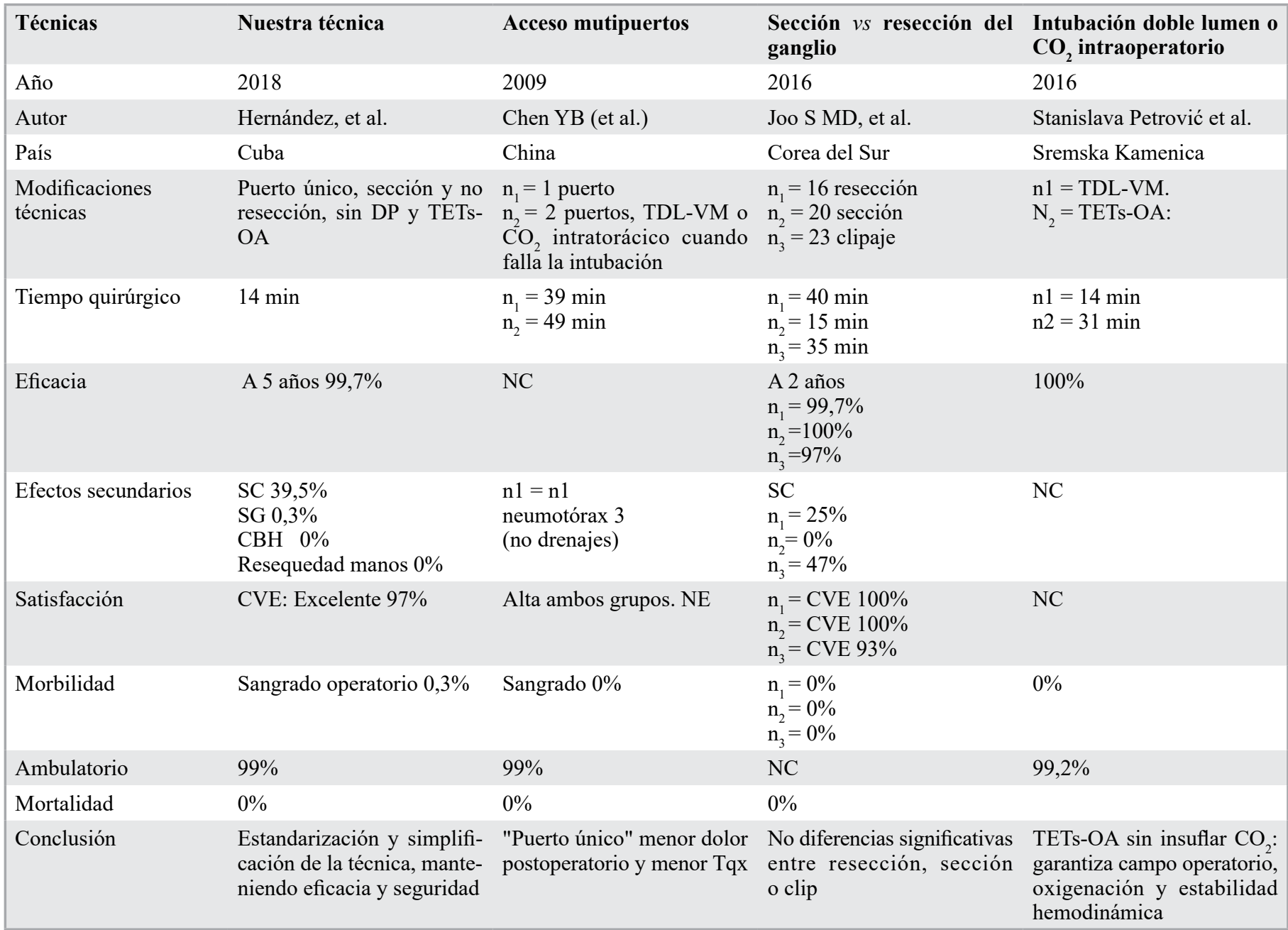

Leyenda: Tqx: Tiempo quirúrgico. DP: Drenajes pleurales; TETs-OA: Anestesia general. Tubo endotraqueal simple con oxigenación apneíca; TDLVM: Tubo de doble luz con ventilación mecánica. SC: Sudor compensatorio; SG: Sudor gustatorio; CBH: Claude Bernard Horner (síndrome); n: Número de pacientes; NC: No conocido; CVE: Calidad de vida excelente.

Tabla 4. Evaluación de ansiedad y depresión según escalas de IDARE, Zung y Conie. Comparativo del inicio vs 5 años del postoperatorio

\begin{tabular}{|c|c|c|c|c|}
\hline \multicolumn{2}{|c|}{$\begin{array}{l}\text { Afectación psíquica (ansiedad, depresión o ambas) } \\
\text { n }\end{array}$} & \multicolumn{2}{|c|}{$\begin{array}{cc} & \text { Total } \\
\% & \text { P valor }\end{array}$} & \\
\hline Preoperatorio & $\begin{array}{l}\text { Leve } \\
\text { Moderado } \\
\text { Severo } \\
\text { Total }\end{array}$ & $\begin{array}{c}51 \\
199 \\
2 \\
252\end{array}$ & $\begin{array}{c}17,1 \\
66,7 \\
0,6 \\
84,5\end{array}$ & \multirow{3}{*}{$\mathrm{p}=0,042546$} \\
\hline 5 Años & $\begin{array}{l}\text { Ninguno } \\
\text { Leve } \\
\text { Moderado } \\
\text { Severo } \\
\text { Total }\end{array}$ & $\begin{array}{c}11 \\
91 \\
150 \\
0 \\
241\end{array}$ & $\begin{array}{c}3,6 \\
30,5 \\
50,3 \\
0 \\
80,8\end{array}$ & \\
\hline Total & & 298 & 100 & \\
\hline
\end{tabular}




\section{Discusión}

Los detalles técnicos aquí descritos se realizan de forma protocolizada en nuestra institución para la simpatectomía por hiperhidrosis, logrando técnica operatoria, simplificada, efectiva y segura para esta entidad. La mayor facilidad la convierte en una técnica reproducible a todos los cirujanos. El empleo de intubación endotraqueal simple permite ventajas bien conocidas sobre el tubo de doble lumen y complementan la estandarización de la simpatectomía para hiperhidrosis palmar. La menor invasión quirúrgica y anestésica redunda en más rápida recuperación y con ella alta precoz, mejor confort y mayor nivel de satisfacción de nuestros pacientes. Son menores el dolor y los procedimientos asociados como drenajes torácicos o antibioticoterapia.

La composición demográfica de nuestro grupo en estudio no tiene diferencia significativa a otras series. Con predominio de pacientes jóvenes, sexo femenino y raza blanca ${ }^{6,12}$. En algunos casos se ha visto tendencia familiar, pero este elemento escapa al interés de la presente revisión.

El transoperatorio se caracterizó por una mayor facilidad anestésica y quirúrgica comparado con series internacionales ${ }^{13-15}$. Como complicación se presentó una pausa sinusal que se instaló bruscamente hasta el paro cardiorrespiratorio, obligando a una reanimación emergente con muy rápida recuperación y sin secuela. Está descrito con baja frecuencia en otras literaturas, independiente al tipo de intubación. Más bien se cree asociado a la simpatectomía ${ }^{16}$. A juicio nuestro es coincidente con pacientes que responden autonómicamente con predominio parasimpático ante distintos estresores como en este caso. Se presentó, además, un sangrado transoperatorio $(0,3 \%)$ y adherencias intratorácicas gruesas en un paciente $(0,3 \%)$. Con la suficiente experiencia se pueden manejar estas situaciones con trocar único, pero la recomendación del presente trabajo es convertir a multipuertos, cirugía abierta o abortar según criterio y experiencia del equipo quirúrgico.

El tiempo quirúrgico es la variable que mayor evidencia aporta en cuanto la simplicidad técnica. Una media de 14 min para ambos hemitórax, mejora notablemente los resultados respecto a series revisadas y a nuestras series anteriores ${ }^{17,18}$.

La estabilidad respiratoria, hemodinámica y de medio interno durante la oxigenación apneica con tubo simple, no varía respecto al paciente ventilado. Está demostrado que aún sin ventilación se produce una adecuada hematosis a nivel de la membrana alveolo capilar ${ }^{19}$. Se logra oxigenación $100 \%$ y buen transporte por un período de 30 a 40 min según la literatura revisada ${ }^{19}$. Para seguridad de nuestros pacientes protocolizamos tiempos máximos de $20 \mathrm{~min}$. De requerir más de este tiempo se acoplaría a ventilación garantizando valores óptimos de oxigenación.

En el periodo inmediato a la cirugía se obtuvo un $100 \%$ de curación. En esta etapa no se presentaron efectos secundarios ni complicaciones siendo el manejo del dolor el único aspecto relevante ${ }^{18-20}$. La totalidad de los pacientes refieren algún grado de dolor. Un gran porcentaje entre moderado y severo (76,9\% entre ambos) según EVA. Además, la tendencia es al aumento en las primeras $72 \mathrm{~h}$, a pesar de no encontrar neumotórax por clínica ni por imagen $^{19}$. A nuestro entender el tratamiento quirúrgico del nervio simpático sobre el periostio de la costilla puede generar estos molestos dolores, en la pared posterior del tórax, por lo que investigaciones posteriores pudieran relacionar estas variables, intentando esclarecer. Importante conocer y orientar correcta educación de los pacientes, medicación oportuna y fisioterapia respiratoria para garantizar un manejo excelente y sin complicaciones.

En el seguimiento a más largo plazo, con corte final a 5 años: las recidivas, efectos secundarios y satisfacción del paciente son las variables universales para evaluar eficacia y seguridad de la técnica.

Solo una paciente presentó recidiva $(0,3 \%)$, debido a reinervación. Ciertamente es la causa descrita en otras publicaciones, pero infrecuente ${ }^{18}$. Nuestra recomendación en este punto va dirigida al nervio de Kuntz. Se debe buscar, exponer y tratar cualquier rama simpática en el trayecto habitual de nervio de Kuntz $^{6}$, y separar quirúrgicamente lo más posible los extremos seccionados.

Los efectos secundarios esperados son variables. La sudoración compensatoria afecta en algún grado a prácticamente al $100 \%$ de los operados. Aparece en el postoperatorio, con más frecuencia en la región de la espalda, cara interior de muslos o glúteos. Aun en presencia de este efecto secundario inevitable, la simpatectomía continúa siendo el tratamiento de elección y único curativo para la hiperhidrosis palmar primaria, según investigaciones nacionales e internacionales ${ }^{20}$.

En el primer corte, a un año, 183 operados $(61,4 \%)$ presentaban sudoración compensatoria moderada, incidencia que disminuyó a los 5 años a 118 pacientes $(39,5 \%)$. La tendencia a disminuir es común en las series revisadas ${ }^{21}$. Es relevante la tolerancia del sudor compensatorio tanto por su topografía como su comportamiento más fisiológico. Aparece en respuesta a calor y ejercicios mientras la hiperhidrosis palmar primaria no respeta mecanismos de control y afecta las herramientas manuales 
con toda su implicación laboral y social. La sudoración compensadora puede ser severa y limitante. Las investigaciones en este sentido orientan al nivel de sección simpático ganglionar ${ }^{22}$. En nuestra serie se presentó en una paciente $(0,3 \%)$ con sudoración severa a nivel de la espalda. Para evitar este fenómeno recomendamos el tratamiento de los niveles 3 y 4 para las manos ${ }^{23}$. Está demostrado que tiene igual eficacia que los niveles 2 y 3 , con menor riesgo de sudor compensador severo.

La sudación gestatoria con 2 pacientes y otras como resequedad de manos son efectos secundarios más raros y su presentación es similar en frecuencia a revisiones internacionales ${ }^{24}$.

No es conocido aún el comportamiento de la sudoración plantar en algunos operados. Aproximadamente el $40 \%$ de los operados por hiperhidrosis palmar, curan o mejoran la variante plantar espontánea y paralelamente. Este beneficio no tiene explicación y no permanece en el tiempo. De la muestra en estudio mejoraron $119(39,9 \%)$ pacientes. A los 5 años solo $52(17,4 \%)$ referían mejoría de la sudación plantar. Ya se cuenta con alguna experiencia en la cirugía de la variedad plantar, pero esto escapa del objetivo de nuestro trabajo.

Tanto la efectividad como los efectos secundarios y nivel de satisfacción de nuestra serie son compatibles con otras series, según estadísticas presentadas en Tabla 4. Se comprende que nuestra simplificación de los aspectos técnicos mantiene la seguridad del paciente y mejora el confort postoperatorio.

Para evaluar nivel de satisfacción para este tratamiento aplicamos encuestas sobre calidad de vida y test validados para ansiedad y depresión, síntomas recurrentes. En el primer caso (Figura 3), el 97\% de nuestros pacientes se encontraron en rangos excelente y bueno a los 5 años. Solo $1 \%$ malo en relación con el sudor compensatorio severo descrito. Estos resultados son significativos y avalan la técnica a pesar de los efectos secundarios como el sudor compensatorio.

Para medir ansiedad y depresión se aplicaron los test de IDARE y la prueba de Zung y Conie respectivamente. Al corte final 102 pacientes habían mejorado sus síntomas respecto estadio preoperatorio. Eso representa mejora de 40,4\%, resultado favorable significativamente, aun cuando estas variables pueden ser multifactoriales.

El presente trabajo es un modelo observacional y no experimental, lo cual limita en si el nivel de evidencia que puede aportar. Sin embargo, los resultados ya conocidos con la intubación doble luz, $\mathrm{CO}_{2}$ para crear campo quirúrgico, empleo de drenajes, multipuertos y otros, contraindican éticamente a modelos experimentalmente entre estas diferentes técnicas quirúrgicas. Entre otras fortalezas, la investigación tiene un tamaño de muestra representativo para nuestra población y la incidencia de la entidad. Fue llevado a cabo por un equipo con experiencia en el tema y con buenos resultados en la técnica. Fueron incluidos sin excepción todos los pacientes que cumplieron 5 años de seguimiento y esto hace una muestra más representativa a nivel poblacional. Aplicamos modelos validados para evaluar variables como calidad de vida. Fue precedido por amplia revisión y actualización bibliográfica. Nuestro equipo tiene investigaciones previas que comparan otras modalidades de tratamiento para esta entidad y conoce resultados con otras variantes de anestesia y cirugía.

\section{Conclusiones}

Minimizar la invasión quirúrgica: puerto único, sección vs resección, menor tiempo quirúrgico y no drenaje pleural, conjuntamente con intubación endotraqueal simple y oxigenación apneíca, permiten

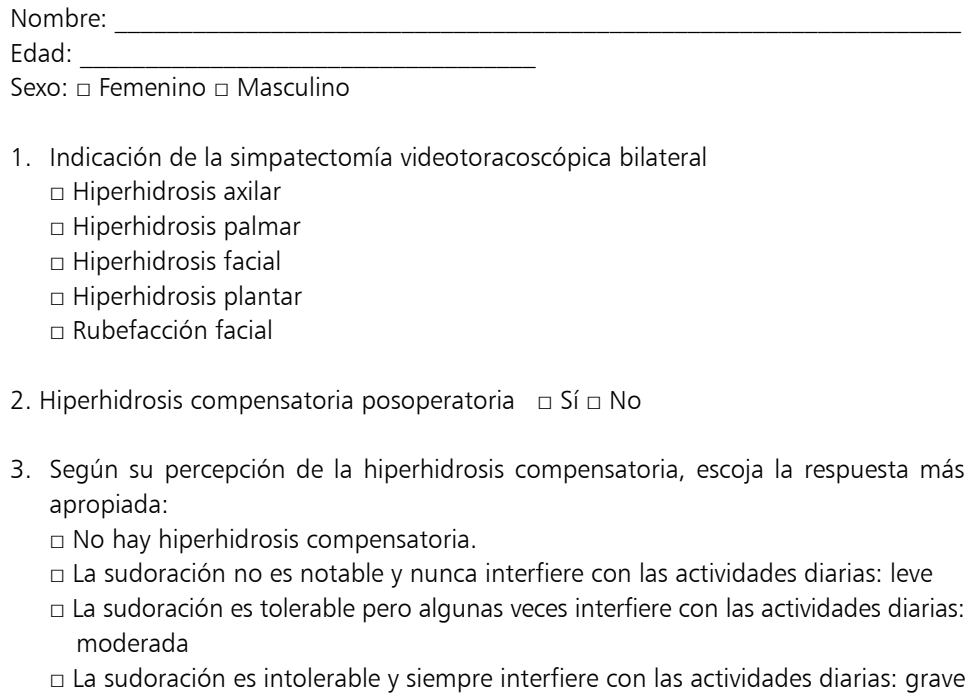

2. Hiperhidrosis compensatoria posoperatoria $\square$ Sí $\square$ No

3. Según su percepción de la hiperhidrosis compensatoria, escoja la respuesta más apropiada:

$\square$ No hay hiperhidrosis compensatoria.

口 La sudoración no es notable y nunca interfiere con las actividades diarias: leve

$\square$ La sudoración es tolerable pero algunas veces interfiere con las actividades diarias: moderada

$\square$ La sudoración es intolerable y siempre interfiere con las actividades diarias: grave

4. Satisfacción personal con los resultados de la cirugía en este momento; califique de 0 a 100:

5. ¿Recomendaría la cirugía a otra persona que presentara sus mismos síntomas? 口 Sí la recomendaría.

$\square$ No la recomendaría

Figura 3. Encuesta sobre calidad de vida (consenso internacional México, 2013). 
menor dolor, más rápida recuperación postoperatoria y manejo ambulatorio siendo una práctica segura para la simpatectomía torácica endoscópica.

Responsabilidades éticas

Protección de personas y animales. Los autores declaran que para esta investigación no se han realizado experimentos en seres humanos ni en animales.

Confidencialidad de los datos. Los autores declaran que en este artículo no aparecen datos de pacientes.

Conflictos de interés: no hay.

\section{Bibliografía}

1. McConaghy JR, Fosselman D. Hyperhidrosis: Management Options. Am Fam Physician. 2018;97:729-34.

2. Martin A, Telford R. Anaesthesia for endoscopic thoracic sympathectomy. Continuing Education in Anaesthesia Critical Care \& Pain 2009;9:52-5.

3. Zamarin J, Egaña J, Berríos R. Calidad de vida en pacientes con hiperhidrosis primaria sometidos a simpatectomía videotoracoscópica. Rev Chil Cir. 2011;63:498-503.

4. Joo S, Lee GD, Haam S, Lee S. Comparisons of the clinical outcomes of thoracoscopic sympathetic surgery for palmar hyperhidrosis: R4 sympathicotomy versus R4 sympathetic clipping versus R3 sympathetic clipping. J Thorac Dis. 2016;8:934-41.

5. Li DC, Hulbert A, Waldbaum B, Ober C, Craig M, Hooker CM, Huang P, et al. Endoscopic thoracic sympathectomy for primary focal hyperhidrosis: impact on psycho-social symptomatology and psychotropic medication use. European Journal of Cardio-Thoracic Surgery 2018;5:1-8. doi.org/10.1093/ejcts/ezy211

6. Cerfolio RJ, Ribas J, Bryant AS, Connery CP, Miller DL, DeCamp MM, et al. The Society of Thoracic Surgeons Expert Consensus for the Surgical Treatment of Hyperhidrosis. Ann Thorac Surg. 2011;91:1642-8.

7. Zhang W, Yu D, Wei Y, Xu J, Zhang $\mathrm{X}$. A systematic review and metaanalysis of T2, T3 or T4, to evaluate the best denervation level for palmar hyperhidrosis. Sci Rep. 2017;7:129. Published online 2017 Mar 9. doi: 10.1038/s41598-017-00169-w

8. Lardinois D, Ris HB. Minimally invasive video-endoscopic sympathectomy by use of a transaxillary single port approach. Eur J Cardiothorac Surg. 2002;21:67-70.
9. Chou SH, Kao EL, Lin CC, Chang YT, Huang MF. The important of classification in sympathethic surgery and proposed mechanism for compensatory hiperhidrosis: experience with 464 cases. Surgical Endoscopy and other interventional techniques 2006;20:174953.

10. Dobosz L, Cwalina N, Stefaniak T. Influence of Body Mass Index on Compensatory Sweating in Patients after Thoracic Sympathectomy due to Palmar Hyperhidrosis. Thorac Cardiovasc Surg. 2017;65:497-502.

11. Chun-An Cheng, Chun-Gu Cheng, Hsin Chu, Hung-Che Lin, Chi-Hsiang Chung, Hung-Wen Chiu. Risk reduction of longterm major adverse cardiovascular events after endoscopic thoracic sympathectomy in palmar hyperhidrosis. Clin Auton Res. 2017;27:393-400.

12. Arroyo T, Giron JC, Loscertales J, Ayarra JE, Arenas C, Congregado M, et al. Tratamiento de la hiperhidrosis palmar por simpatectomía torácica. Resultados inmediatos y calidad de vida postoperatoria. Archivos de Bronconeumología 2004;40:67-71.

13. Weng W, Liu Y, Li J, Jiang G, Liu J, Wang J. A long-term evaluation of postoperative moist hands after R4/ R4+5 sympathectomy for primary palmar hyperhidrosis. J Thorac Dis. 2018;10:1532-7.

14. Ibrahim M, Menna $C$, Andreetti $C$, Ciccone AM, D'Andrilli A, Maurizi G, et al Two-stage unilateral versus one-stage bilateral single-port sympathectomy for palmar and axillary hyperhidrosis. Interact Cardiovasc Thorac Surg. 2013;16:834-8.

15. Aydemir B, Imamoglu O, Okay T, Celik M. Sympathectomy versus Sympathicotomy in Palmar Hyperhidrosis Comparing T3 Ablation. Thorac Cardiovasc Surg. 2015;63:715-9.
16. Zhang W, Wei Y, Jiang H, Xu J, Yu D. R3 versus R4 Thoracoscopic Sympathectomy for Severe Palmar Hyperhidrosis. Thorac Cardiovasc Surg. 2017;65:491-6.

17. Chen YB, Ye W, Yang WT, Shi L, Guo $\mathrm{XF}, \mathrm{Xu} \mathrm{ZH}$, et al. Uniportal versus biportal video-assisted thoracoscopic sympathectomy for palmar hyperhidrosis. Chin Med J Engl. 2009;122:1525-8.

18. Torng-Sen Lin, Shou-Jen Kuo, Ming-Chih Chou. Uniportal Endoscopic Thoracic Sympathectomy for Treatment of Palmar and Axillary Hyperhidrosis: Analysis of 2000 Cases. Neurosurgery 2002;51:84-7.

19. Nodal J, Olivé JB, Machado M, Quintana I. Utilización de la oxigenación apneica en la simpatectomía transtorácica endoscópica. Revista Cubana de Anestesiologìa y Reanimación 2010;9:200-10.

20. Montessi J, Pereira E, Vieira JP, da Matta M, Passos RL, Duarte OV. Video-assisted thoracic sympathectomy in the treatment of primary hyperhidrosis: a retrospective study of 521 cases comparing different levels of ablation. J Bras Pneumol. 2007;33:248-54

21. Mark J. Krasna, MD. Thoracoscopic Sympathectomy: A Standardized Approach to Therapy for Hyperhidrosis. Ann Thorac Surg. 2008;85:764-7

22. Kesler KA, Brooks-Brunn J, Campbell RL, Brown JW. Thoracoscopic Sympathectomy for Hyperhidrosis Palmaris: A periareolar Approach. Ann Thorac Surg. 2000;70:314 -7.

23. Zhang W, Wei Y, Jiang H, Xu J, Yu D. T3 versus $\mathrm{T} 4$ thoracoscopic sympathectomy for palmar hyperhidrosis: a meta-analysis and systematic review. J Surg Res. 2017;218:124-31.

24. Sang HW, Li GL, Xiong P, Zhu MC, Zhu M. Optimal targeting of sympathetic chain levels for treatment of palmar hyperhidrosis: an updated systematic review. Surg Endosc. 2017;31:4357-69. 